\title{
Numerical prediction of pressure pulsation amplitude for different operating regimes of Francis turbine draft tubes
}

\author{
Andrej Lipej, Dragica Jošt, Peter Mežnar and Vesko Djelić \\ Turboinštitut d.d. \\ Rovšnikova 7, Ljubljana, 1210, Slovenia, \\ andrej.lipej@turboinstitut.si
}

\begin{abstract}
Hydraulic instability associated with pressure fluctuations is a serious problem in hydraulic machinery. Pressure fluctuations are usually a result of a strong vortex created in the centre of a flow at the outlet of a runner. At every radial turbine and also at every single regulating axial turbine, the draft tube vortex appears at part-load operating regimes. The consequences of the vortex developed in the draft tube are very unpleasant pressure pulsation, axial and radial forces and torque fluctuation as well as turbine structure vibration. The consequences of the vortex are transferred upstream and downstream with amplitude and frequency modulation in respect of the turbine operating regime, cavitation conditions and air admitted content.
\end{abstract}

Numerical prediction of the vortex appearance in the design stage is a very important task. The amplitude of the pressure pulsation is different for each operating regime therefore the main goal of this research was to numerically predict pressure pulsation amplitude versus different guide vane openings and to compare the results with experimental ones.

For the numerical flow analysis of a complete Francis turbine (FT), the computer code ANSYS-CFX11 has been used.

Keywords: radial turbine, vortex, pressure pulsation.

\section{Introduction}

The computational grids are prepared with ANSYS-ICEM Hexa and TurboGrid meshes. The computational grids of the Francis turbine (Fig. 1) consist of from 3.3 to 25 million elements for different cases.

The first part of the paper presents the numerical method for prediction of a vortex rope in the draft tube of a Francis turbine using unsteady flow simulations.

The second part deals with the analysis of unsteady calculation of vortex rope prediction and pressure pulsation in the draft tube. The time steps for unsteady calculations were adequate from one to six degrees of the runner revolution. The computation for each operating regime was running for more than ten complete runner revolutions. The computational time for a single operating point depends on the convergence rate and the number of processors used in the computer cluster. The first calculations were done on the computer cluster with 32 dual-core processors.

For other analyses, a new supercomputer cluster at Turboinštitut with 2048 cores was used. In the third part, the results of numerical analyses and results obtained on the model tests are compared for all operating regimes. 


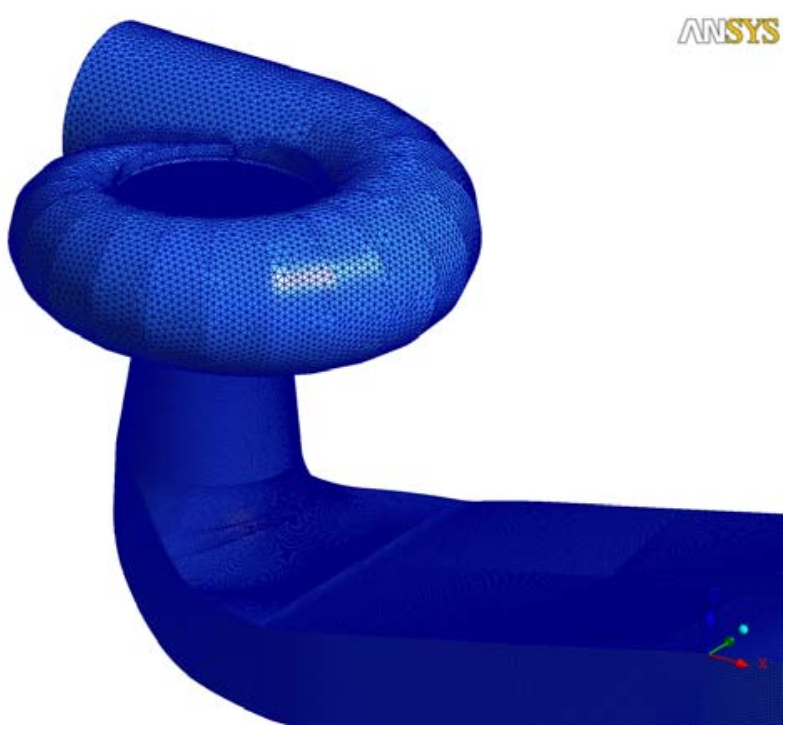

Fig. 1 Computational grid for Francis turbine

\section{Numerical method}

\subsection{Basic equations}

Basic equations: continuity and momentum [1]

$$
\begin{aligned}
& \frac{\partial \rho}{\partial t}+\nabla \bullet(\rho U)=0 \\
& \frac{\partial(\rho U)}{\partial t}+\nabla(\rho U \otimes U)=-\nabla p+\nabla \bullet \tau+S_{M}
\end{aligned}
$$

For flows in a rotating frame of reference, rotating at a constant angular velocity, additional sources of momentum are required to account for the effects of the Coriolis force and the centrifugal force:

$S_{M, r o t}=S_{C o r}+S_{c f g}$

where Coriolis force is presented by

$S_{\text {Cor }}=-2 \rho \omega \times U$

and centrifugal force is equal to

$S_{c f g}=-\rho \omega \times(\omega \times r)$

Turbulence models are used to predict the effects of turbulence in fluid flow without resolving all scales of the smallest turbulent fluctuations. A number of models can be used to approximate turbulence based on the Reynolds Averaged NavierStokes (RANS) equations. Some have been developed for very specific applications while the others can be applied to a wider class of flows with a reasonable degree of confidence. The models can be classified as either eddy-viscosity or Reynolds stress models.

One of the most prominent turbulence models, the $\mathrm{k}-\varepsilon$ (k-epsilon) model, has been implemented in most of the general purpose CFD codes and is considered the industry standard model. It has proven to be stable and numerically robust and has a well established regime of predictive capability. For general purpose simulations the k- $\varepsilon$ model offers a good compromise in terms of accuracy and robustness.

Standard two-equation models, such as the k- $\varepsilon$ model, provide good predictions for many flows of engineering interest. There are some applications for which these models may not be suitable. Among these are: flows with boundary layer separation, flows with sudden changes in the mean strain rate, flows in rotating fluids and flows over curved surfaces. Reynolds Stress models may be more appropriate for flows with sudden changes in strain rate or rotating flows while the SST model may be more appropriate for separated flows. The SST model is recommended for high accuracy boundary layer simulations. The convergence behaviour of the $\mathrm{k}-\omega$ model is often similar to that of the $\mathrm{k}-\varepsilon$ model. 
In flows where the turbulent transport or non-equilibrium effects are important, the eddy-viscosity assumption is no longer valid and results of eddy-viscosity models might be inaccurate. Reynolds Stress models naturally include the effects of streamline curvature, sudden changes in the strain rate, secondary flows or buoyancy compared to turbulence models using the eddyviscosity approximation. Theoretically, Reynolds Stress models are more suited to complex flows, however, practice shows that they are often not superior to two-equation models. If convergence is difficult, it is recommended that a k- $\varepsilon$ or k- $\omega$ based model solution should be obtained first and then a Reynolds stress model solution can be attempted from the converged two-equation solution. It is frequently observed that Reynolds Stress models produce unsteady results, where two-equation models give steady state solutions. This can be physically correct, but requires the solution of the equations in a transient mode.

For the transient calculation it is possible to use Unsteady Reynolds Averaged Navier-Stokes (URANS) models but the URANS simulation produces only the large-scale unsteadiness. The Scalable Adaptive Simulation (SAS) Shear Stress Transport (SST) method is an improved URANS formulation, with the ability to adapt the length scale to resolved turbulent structures. The SAS concept is based on the introduction of the von Karman length scale into the turbulence scale equation. The information provided by the von Karman length scale allows SAS models to dynamically adjust to resolved structures in a URANS simulation, which results in a Large Eddy Simulation (LES)-like behaviour in unsteady regions of the flow field. At the same time, the model provides standard RANS capabilities in stable flow regions. The SAS-SST model also adjusts to the already resolved scales in a dynamic way and allows the development of a turbulent spectrum in the detached regions.

\subsection{SAS-SST Model Formulation}

The starting point of the transformation to the SST model is the $k-v$, formulation as given by Menter and Egorov [2]. The following equations have been derived there for the variables $\mathrm{k}$ and $\Phi=\sqrt{ } k L$ :

$$
\begin{aligned}
& \frac{\partial \rho k}{\partial t}+\frac{\partial U_{j} \rho k}{\partial x_{j}}=P_{k}-c_{\mu}^{\frac{3}{4}} \rho \frac{k^{2}}{\Phi}+\frac{\partial}{\partial y}\left[\frac{\mu_{t}}{\sigma_{k}} \frac{\partial k}{\partial y}\right] \\
& \frac{\partial \rho \Phi}{\partial t}+\frac{\partial U_{j} \rho \Phi}{\partial x_{j}}=\zeta_{1} \frac{\phi}{k} P_{k}-\zeta_{2} \mu_{t} S\left|U^{\prime \prime}\right| \frac{\Phi^{2}}{k^{\frac{3}{2}}}-\zeta_{3} \rho k+\frac{\partial}{\partial y}\left[\frac{\mu_{t}}{\sigma_{\Phi}} \frac{\partial \Phi}{\partial y}\right] \\
& v_{t}=c_{\mu}^{\frac{1}{4}} \Phi
\end{aligned}
$$

with

$$
\left|U^{\prime \prime}\right|=\sqrt{\frac{\partial^{2} U_{i}}{\partial x_{j}^{2}} \frac{\partial^{2} U_{i}}{\partial x_{j}^{2}}} .
$$

$S$ is the absolute value of the strain-rate, $P_{k}$ is the production rate of the turbulent kinetic energy, and the values of constants are: $\mathrm{c}_{\mathrm{m}}=0.09, \quad \mathrm{k}=0.41$,

$\zeta_{1}=0.8, \quad \zeta_{2}=3.51, \quad \zeta_{3}=0.0326$.

The SAS-relevant term in the equation for is the term with the second derivative |U"|. As a result of this term, the length scale L predicted by the above model is largely proportional to the von Karman length scale:

$$
L_{v K}=\left|\frac{\frac{\partial U}{\partial y}}{\frac{\partial^{2} U}{\partial y^{2}}}\right| .
$$

In this paper, the SAS-SST turbulent model is used for a prediction of the vortex rope in the draft tube. This model is mostly used for CFD simulations; where steady-state simulations are not of sufficient accuracy and do not properly describe the true nature of the physical phenomena. For problems with strong mixing behind blades and analysis of vortex rope in draft tubes, the SAS-SST model provides a more accurate solution than (URANS) models.

The Transient Rotor-Stator model should be used any time when it is important to take into account transient interaction effects at a sliding (frame change) interface. It predicts the true transient interaction of the flow between a stator and a rotor passage. It ultimately accounts for all interaction effects between components that are in relative motion to each other. The interface position is updated at each time step, as the relative position of the grids on each side of the interface changes. The principle disadvantage of this method is that the computer resources required are large, in terms of simulation time, disk space and quantitative post-processing of the data. It is possible to start a transient rotor-stator computation from a simple initial guess, or from an existing prediction.

When we are not interested in the start-up transient of the machine we can start from the steady state sliding interface solution 
using Frozen Rotor interfaces between components.

\section{Numerical prediction of vortex rope}

For part-load conditions, the vortex (also called rope) spirals outward and processes in the direction of the turbine's rotation typically between one fourth and one third of the turbine's rotational frequency. These phenomena can cause large pressure fluctuations, low frequency vibrations and undesirable variations in turbine output. In this paper, the numerical prediction of the frequency and amplitudes of pressure pulsation caused by vortex rope is presented.

\subsection{Computational grids}

For the numerical analyses, different computational grids and domain configurations were used (Table 1). First the computational grid of the complete Francis turbine including spiral casing with stay vanes, guide vane cascade, runner and draft tube with 3,300,000 elements was used. The second computational grid of the complete Francis turbine consists of the total $17,000,000$ elements. The third configuration consists of runner and draft tube with 25,000,000 elements. The grid of the spiral casing is made using tetrahedral elements with prism layers near the walls, all other turbine parts are discretized by hexahedral elements.

Table 1 Number of elements in particular geometry configuration

\begin{tabular}{|c|c|c|c|r|}
\hline & $\begin{array}{c}\text { Spiral casing, } \\
\text { stay and guide vanes }\end{array}$ & Runner & Draft tube & Total \\
\hline Configuration 1 & $1,400,000$ & $1,000,000$ & 900,000 & $3,300,000$ \\
\hline Configuration 2 & $1,400,000$ & $12,600,000$ & $3,000,000$ & $17,000,000$ \\
\hline Configuration 3 & - & $12,600,000$ & $12,400,000$ & $25,000,000$ \\
\hline
\end{tabular}

The length of the time step in unsteady calculation was equal to one, three or six degrees of the runner revolution and the time steps were $\mathrm{t}_{\mathrm{o}}=1.810^{-4} \mathrm{~s}, \mathrm{t}_{\mathrm{o}}=5.410^{-4} \mathrm{~s}$ and $\mathrm{t}_{\mathrm{o}}=1.0810^{-3} \mathrm{~s}$ respectively. Convergence criteria was prescribed to $510^{-5}$ for RMS and in the average a converged solution was obtained after three to ten iterations in each time step, depending on the flow rate operating regime.

\subsection{High performance computing facilities}

First mesh configuration was analyzed by the old computer cluster with 32 dual core processors. For the second and third configurations a new supercomputer cluster with 2048 processor cores - 512 Quad-Core Intel Xeon processors L5335 - was used. The cluster (Fig. 2) has in total more than 2 TB RAM and for high performance computing communications InfiniBand is used. The supercomputer was installed at Turboinštitut in May 2008 and because of a short of time availability, only a limited number of calculations have already been done.

The CPU time for one complete runner revolution using the old computer was about 24 hours on eight processors.

At the beginning of using the new computer cluster the speed-up for different numbers of processors and different grid configurations was analyzed. The total computational time for the second grid configuration with 17 million elements using the new computer cluster was about ten hours for one runner revolution when using 32 quad-core processors (128 cores). For computational grids with the order of magnitude about 20 million elements, the maximum number of processors was 64 quad-core processors (256 cores) because at higher numbers the speed-up is negligible. Special attention has to be focused on the number of elements in a particular domain because of the partitioning process.
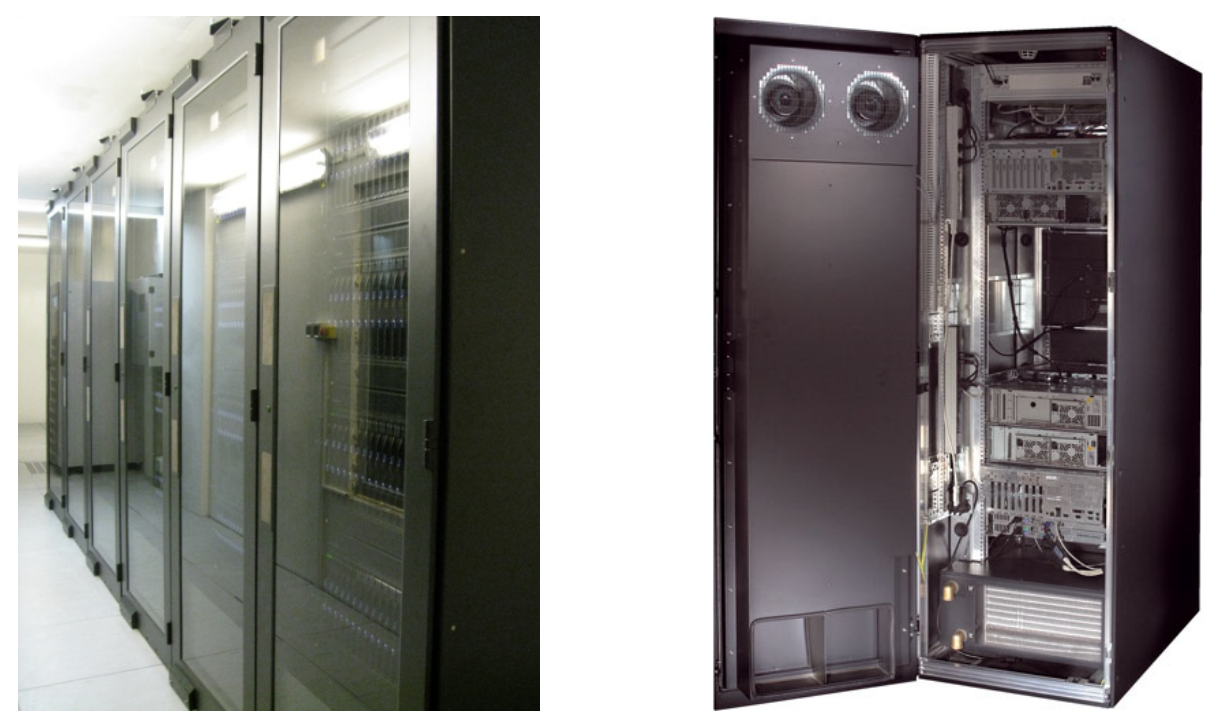

Fig. 2 Supercomputer installed at Turboinštitut six water cooled racks (left), back view of the water cooled rack (right) 
The software has not been developed for running on the huge number of processors yet. That is why all analyses have been done on 64,128 or 256 cores.

\section{Model test}

The main reason for appearance of the vortex rope in the draft tube is the non-uniform velocity distribution near the trailing edge of the runner blades. In the Fig. 3 the axial velocity component as a function of radius is presented at the inlet of the draft tube for all analyzed operating points. It is obvious that at $\mathrm{Ao}=1.25$, where the velocity distribution is almost constant, the pressure pulsations are negligible.

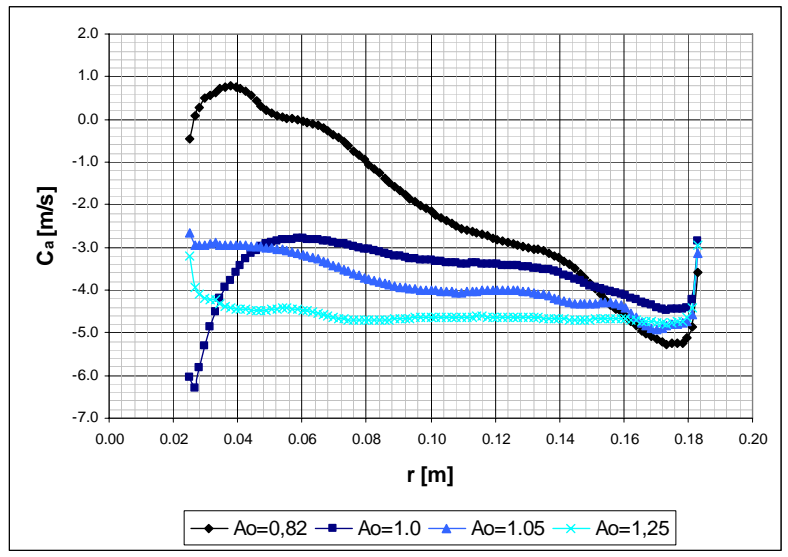

Fig. 3 Axial velocity component behind the runner for different operating regimes

Pressure fluctuations on a Francis model turbine at Turboinštitut are observed and measured in accordance with IEC 90193. For this purpose KISTLER piezoresistive absolute pressure transducers are located on different locations on the pressure and suction side. An example of transducer positions is presented in Fig. 4. The main purpose of these measurements was to obtain enough information about the magnitude and nature of pressure fluctuations, their dominant frequency and additionally the dampening effect of air admission.

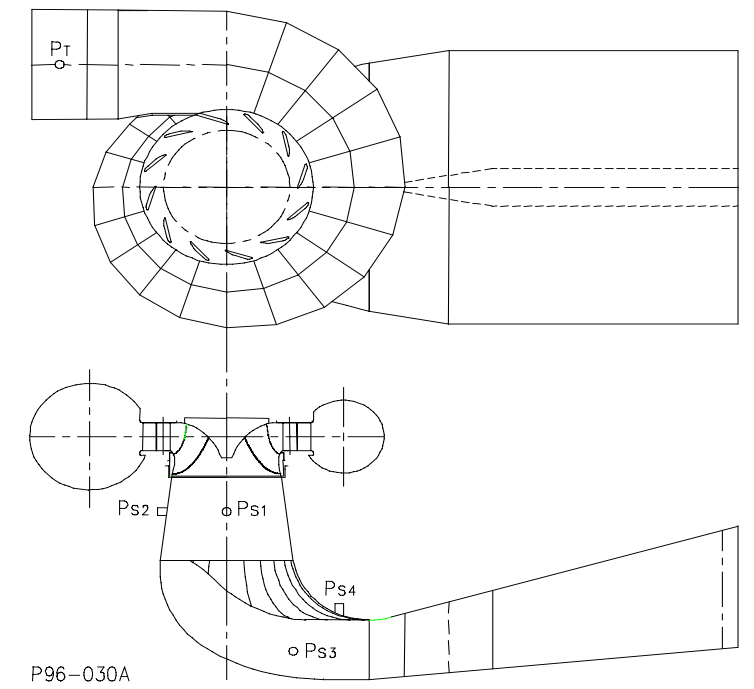

Fig. 4 Location of pressure transducers

Signals from the pressure transducers are wired to the multichannel data acquisition system, based on National Instruments PCI-MIO-16XE-10 multifunction card with additional SCXI signal conditioning modules. Signals are acquired continuously with $5 \mathrm{KHz}$ sampling frequency and 16 bit resolution. Binary data samples stored on the computer hard disk are at least 30 seconds long. Software developed in LabVIEW is used to record and analyze pressure signals (Fig. 5).

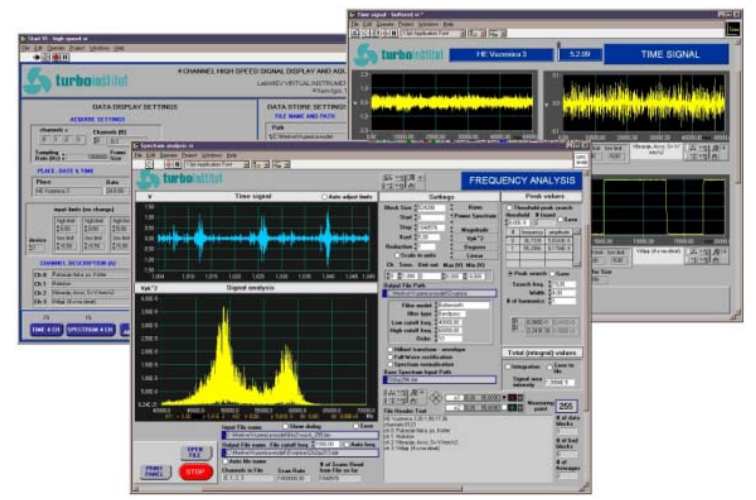


Fig. 5 Data acquisition and processing software based on LabVIEW environment

\section{Results of pressure pulsations}

The numerically predicted frequency of the vortex rope can be obtained from the analysis of pressure distribution on the cross section under the runner at different time steps. The positions of the centre of a low pressure zone (Fig. 6) at different time steps are determined and the angle between the obtained points can be measured.

The numerically obtained frequency of the vortex rope is about $20 \%$ to $26 \%$ of the runner rotational speed depending on the operating regime.
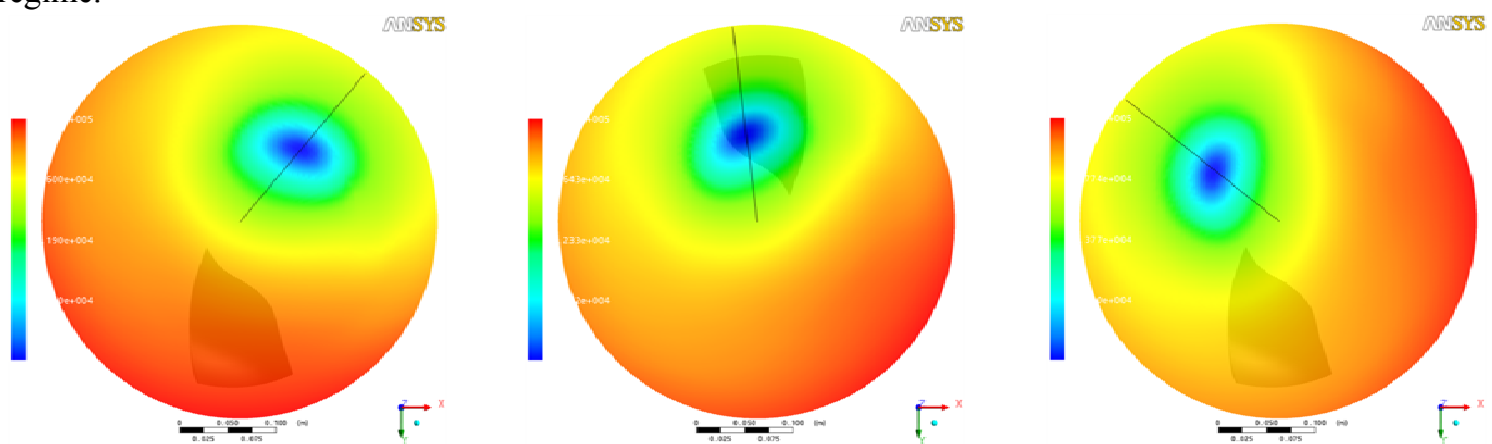

Fig. 6 Location of low pressure zone in draft tube cone cross section during one runner revolution

The frequency spectrum obtained from the measurements on the model test rig is very similar to the numerically obtained values.
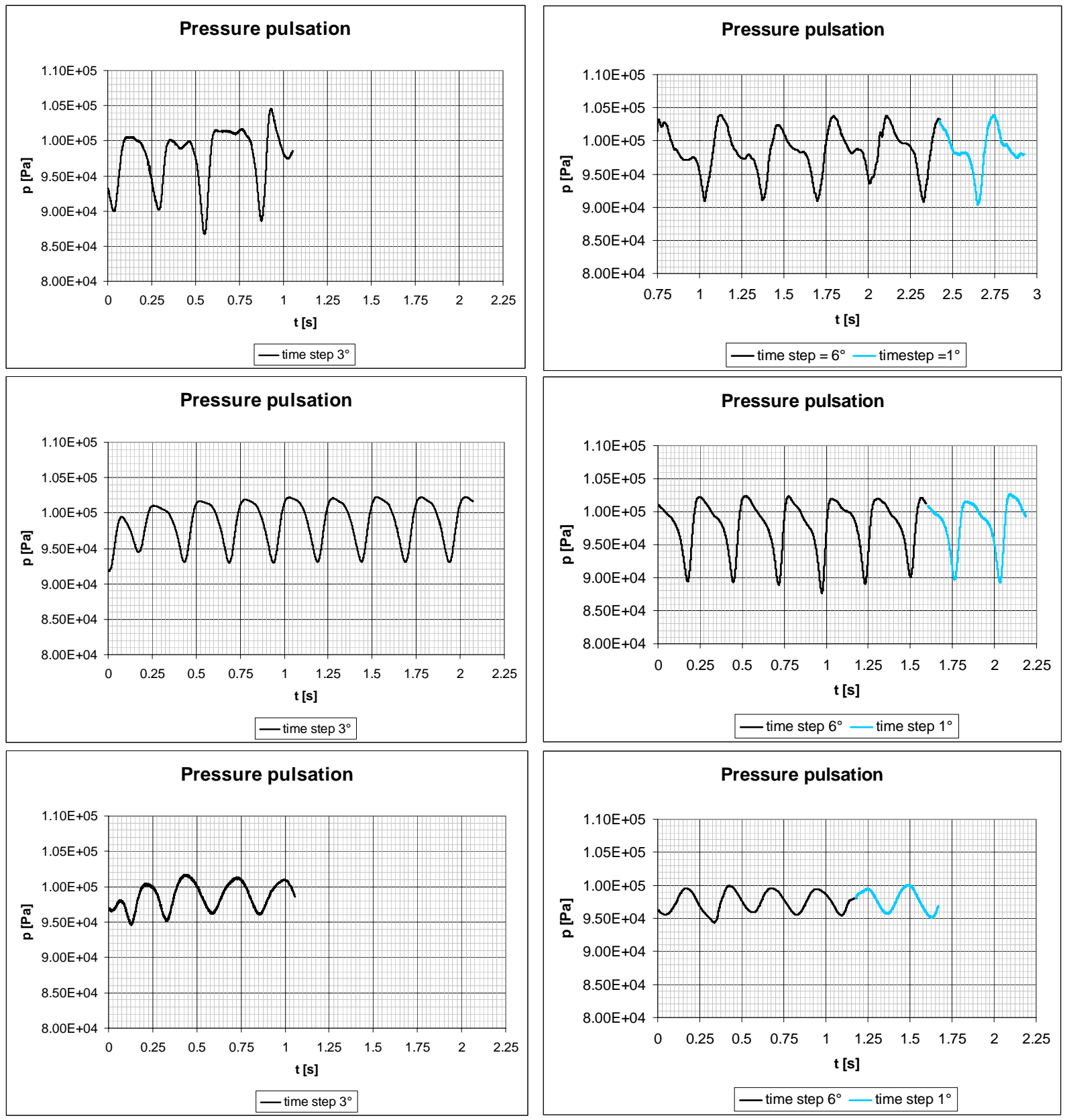
Fig. 7 Pressure pulsations for three operating points: $\mathrm{A}_{\mathrm{o}}=0.82, \mathrm{~A}_{\mathrm{o}}=1.0, \mathrm{~A}_{\mathrm{o}}=1.05$ (downwards) and for two computational grids: $\mathrm{a}-3.3 \mathrm{M}$ elements, $\mathrm{b}-17 \mathrm{M}$ elements

The difference between numerical and experimental results is about $1 \%$ of the runner rotational speed. In Fig. 7 the pressure distributions at the location PS2 (defined in Fig. 4) are presented.

Beside the vortex rope frequency, the main challenge of the numerical analysis was the prediction of the pressure pulsation amplitudes for different operating regimes. The analysis started with an optimal operating point, where the pressure pulsation amplitudes are very low, and proceeded with lower flow rates $\mathrm{Q} / \mathrm{Q}_{\mathrm{bep}}=0.8$. Calculations were done also for $\mathrm{Q} / \mathrm{Q}_{\mathrm{bep}}=0.76$ where the amplitude has maximal value and for an operating regime at the lowest flow rate $\mathrm{Q} / \mathrm{Q}_{\mathrm{bep}}=0.62$, where the pressure amplitude drops down again. The comparison between the measurements and the numerical results shows that numerical prediction of the position of maximal pressure pulsation amplitude is quite accurate, but the values of the amplitudes are lower in comparison with the experimental results except for the operating regime with the smallest flow rate.

Graphs presented in Fig. 7 show pressure pulsations for two different grid configurations. For the coarse grid (a) the time step is $3^{\circ}$ of runner revolution. For the fine grid with $17 \mathrm{M}$ elements (b) the first part of the particular graph shows the distribution of the pressure for time step $6^{\circ}$ and the last part for the time step equal to $1^{\circ}$. For this case the differences in frequency and amplitude due to the time step are negligible. The vortex rope frequency is, at the beginning of the calculation, lower (Fig. 9) than the frequency obtained from the measurements (Fig. 8). After five complete vortex revolutions the numerical and experimental values of the frequency differ by about one percent. In Fig. 9 the history of the frequency during the calculation is shown for two operating regimes and compared to the measured values.

From the analysis of the pressure pulsations amplitudes it can be seen that with coarse computational grids - $3.3 \mathrm{M}$ elements the difference between numerical results and experimental results can be more than $50 \%$ in an operating regime with maximal amplitude. With a finer computational grid - $17 \mathrm{M}$ elements - the accuracy is better and the difference is less than 10\% (Fig. 10).
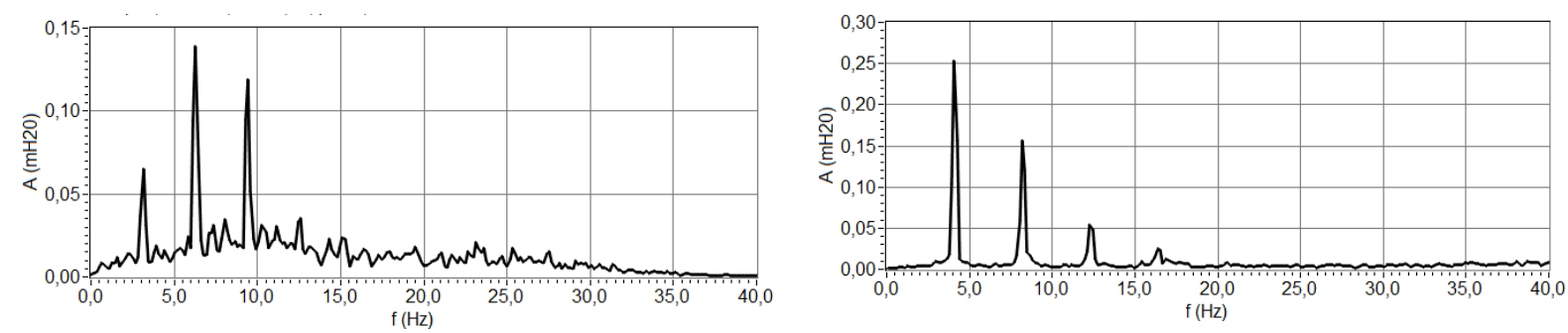

Fig. 8 Vortex rope frequency for two operating points; $\left(A_{0}=0,82-\right.$ left, $A_{0}=1.0$ - right $)$, experimental results

The analysis of the pressure pulsation amplitudes was done just for one head, because the computations are time consuming. The analyses for more heads are planned to be done in the future.

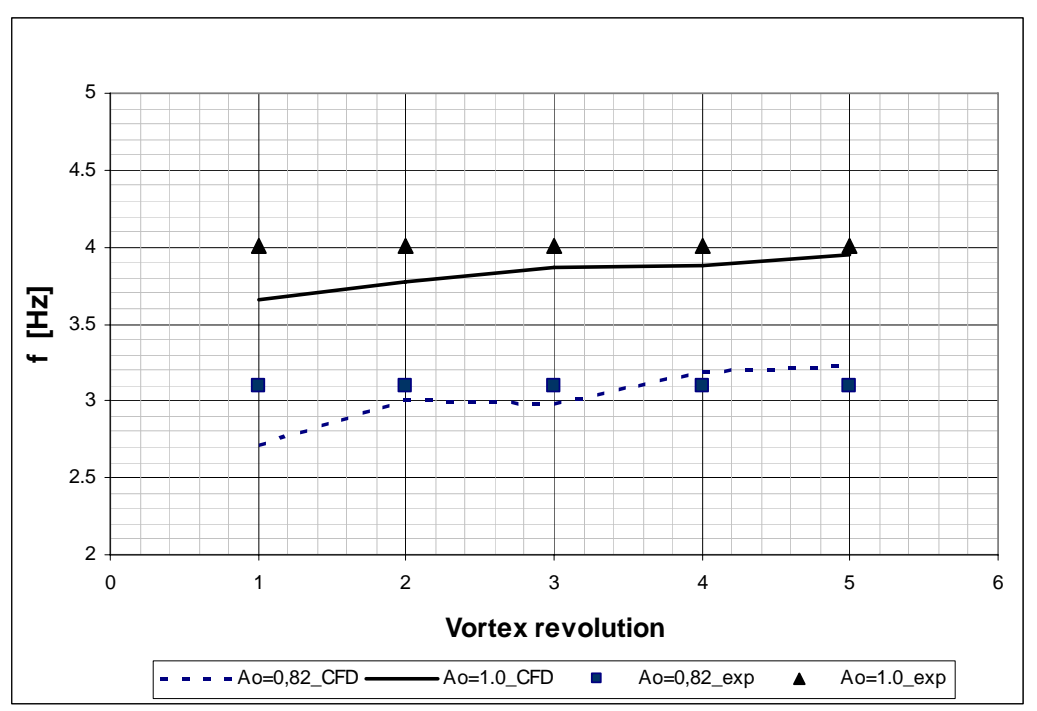

Fig. 9 Vortex rope frequency variation during the calculation for two relative guide vane openings compared to experimental values

The significant difference is obtained in pressure pulsation amplitudes for two different computational grids (Fig. 7). The detailed results and comparison with measurements are presented in Fig. 10. 


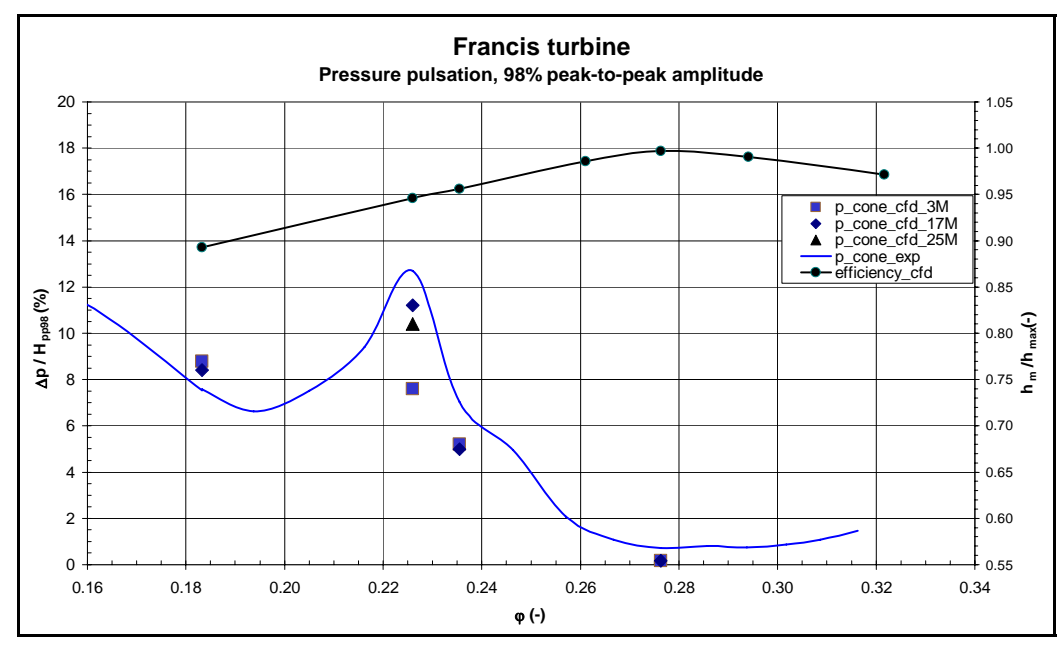

Fig. 10 Pressure pulsation amplitudes for different operating regimes; comparison between experimental and numerical values

\section{Conclusion}

Nowadays powerful supercomputers enable us to analyze different unsteady phenomena in turbo machinery. Accurate results can be obtained using quality computational grids, the appropriate turbulence model and an unsteady numerical model with adequate time steps to resolve all details in the flow. In the paper the numerical and experimental results of the pressure pulsations in the draft tube of a Francis turbine were presented.

The comparison between numerical and experimental analyses of the pressure pulsation in the draft tube of a Francis turbine shows satisfactory agreement for almost all operating regimes. First of all the unsteady analysis is necessary. Secondly, the converged results can be obtained by the calculation using sufficient time steps and appropriate length of the single time step. The minimum number of time steps has to be adequate to four vortex revolutions and the length of a single time step can be six degrees of the runner revolution. Because the rotational speed of the vortex is usually from $20 \%$ to $35 \%$ of the runner speed, reliable results can be obtained after twelve to sixteen runner revolutions. Finally, it is important to choose a suitable turbulence model which enables us to obtain the result in a reasonable computational time. The SAS-SST was found as a suitable turbulence model for industrial applications.

The comparison of the vortex frequency and the pressure pulsation amplitudes shows that the experimentally and numerically obtained frequencies are very close, while the prediction of amplitudes is less accurate.

\section{Acknowledgments}

The research is partially financed by the Slovenian Research Agency ARRS - Contract no L2-1067-0263-08.

\section{Nomenclature}

$\begin{array}{ll}f & \text { Frequency }\left[\mathrm{s}^{-1}\right] \\ k & \text { Turbulent kinetic energy }\left[\mathrm{m} 2 \mathrm{~s}^{-2}\right] \\ L & \text { Length scale }[\mathrm{m}] \\ S & \text { Strain rate }\left[\mathrm{s}^{-1}\right]\end{array}$

$f \quad$ Frequency $\left[\mathrm{s}^{-1}\right]$

Length scale $[\mathrm{m}]$

$\begin{array}{ll}T & \text { Local mean temperature }[\mathrm{K}] \\ U_{i} & \text { Velocity components }\left[\mathrm{ms}^{-1}\right] \\ \phi & \text { Flow rate coefficient }[-] \\ \rho & \text { Fluid Density }\left[\mathrm{kgm}^{-3}\right]\end{array}$

\section{References}

[1] ANSYS CFX, 2005, Release 10, Documentation.

[2] Menter F. R. and Egorov, Y., 2004, "Re-visiting the turbulent scale equation," Proc. IUTAM Symposium; One hundred years of boundary layer research, Göttingen. 